\title{
CARACTERÍSTICAS SENSORIAIS DA CARNE DE CORDEIRO MATURADA E INJETADA COM CLORETO DE CÁLCIO*
}

\author{
SENSORY CHARACTERISTICS OF LAMB MEAT AGEING AND INJECTED \\ WITH CALCIUM CHLORIDE
}

\begin{abstract}
Zeola, N.M.B.L. ${ }^{1 A}$, Souza, P.A. ${ }^{1 B}$, Souza, H.B.A. ${ }^{1 C}$ e Silva Sobrinho, A.G. ${ }^{1 D}$
${ }^{1}$ Faculdade de Ciências Agrárias e Veterinárias (FCAV). Unesp, Via de Acesso Prof. Paulo Donato Castellane, s/n. Jaboticabal-SP. Brasil. Anivea.brancacci@ig.com.br; Bpasoz@fcav.unesp.br; chiras@fcav.unesp.br; Damerico@fcav.unesp.br
\end{abstract}

\section{PaLAVRAS ChaVE ADICIONAIS}

Análise sensorial.

\section{RESUMO}

Foram estudadas as características sensoriais da carne de cordeiros Morada Nova proveniente dos músculos Biceps femoris, Longissimus e Triceps brachii submetidos aos efeitos do tempo de maturação e da injeção com cloreto de cálcio. Os cordeiros foram abatidos ao atingirem $25 \mathrm{~kg}$ de peso vivo. Após o período de instalação do rigor mortis, os músculos foram retirados e submetidos aos respectivos tratamentos. A análise de variância indicou que o tempo de maturação e o fator cloreto de cálcio não influenciaram os atributos sabor $(6,71)$ e maciez $(7,13)$ das carnes provenientes do Biceps femoris. A interação entre os fatores foi não significativa para o atributo maciez, entretanto foi significativa para o atributo sabor. O tempo de maturação não afetou os atributos cor $(7,08)$, sabor $(7,42)$, maciez $(7,83)$ e impressão global $(7,58)$ das carnes provenientes do Longissimus sem cloreto de cálcio, para as carnes com cloreto de cálcio estes atributos também não foram afetados. O tempo de maturação e o cloreto de cálcio não influenciaram os atributos sabor $(6,88)$ e maciez $(7,34)$ das carnes provenientes do Triceps brachii. A interação entre os fatores foi não significativa para os atributos sabor e maciez. A qualidade sensorial da carne de

*Parte da Tese de Doutorado do primeiro autor, Projeto parcialmente financiado pela FUNDUNESP e pelo Programa de Pós-Graduação em Zootecnia da FCAV-UNESP.

Recibido: 27-10-05. Aceptado: 4-2-09.
AdDitionAL KEYWORDS

Sensory evaluation.

cordeiros Morada Nova, mostrou-se semelhante quanto aos atributos avaliados quando fo submetida aos processos de maturação e injeção com cloreto de cálcio.

\section{SUMMARY}

Sensory characteristics of Morada Nova lambs meat (muscles Biceps femoris, Longissimus and Triceps brachii) submitted to effects of ageing and injection of calcium chloride were studied. The lambs were slaughtered at $25 \mathrm{~kg}$ of body weight. After rigor mortis, muscles were removed and submitted to treatments. The variance analysis indicated that ageing and calcium chloride didn't influence the attributes flavor (6.71) and tenderness (7.13) of meats from Biceps femoris. The interaction among the factors wasn't significant for tenderness, however it was significant for flavor. Ageing didn't affect colour (7.08), flavor (7.42), tenderness (7.83) and global impression (7.58) of meats from Longissimus without calcium chloride, and meats with calcium chloride also didn't affect. Ageing and calcium chloride factor didn't influence the attributes flavor and tenderness of meats from Triceps brachii. The interaction among the factors wasn't significant for attributes flavor and tenderness. The sensory quality of Morada Nova lambs meat, was shown similar with relationship to evaluated attributes when it was submitted to ageing and injection with calcium chloride.

Arch. Zootec. 59 (228): 539-548. 2010. 


\section{INTRODUÇÃO}

A análise sensorial é uma ferramenta utilizada na tecnologia de alimentos que serve para medir, analisar e interpretar as reações produzidas pelas características dos alimentos da forma que são percebidas pelos órgãos da visão, odor, gosto, tato e audição (Silva, 2002). Os métodos sensoriais dividem-se em três grupos: métodos de diferença (teste triangular, teste duo-trio, teste de comparação pareada, teste de ordenação e teste de diferença do controle), métodos descritivos (perfil de sabor, perfil de textura e ADQ ou análise descritiva quantitativa) e métodos afetivos (teste de comparação pareada, teste de ordenaçãopreferência e teste de escala hedônica) (Osório et al., 1998). A palavra hedônica refere-se aos estados psicológicos conscientes agradáveis ou desagradáveis. $\mathrm{O}$ objetivo do teste de aceitação é avaliar o grau com que o provador gosta ou desgosta de um produto.

Muitas pesquisas no Brasil foram realizadas nas últimas décadas a respeito das características da carne de cordeiro, porém, a maioria destas foram baseadas em métodos instrumentais e muito poucas em análise sensorial (Martínez-Cerezo et al., 2005). De acordo com Vilas Boas (2005) no Brasil não há ainda estudos expressivos sobre a aceitação comercial da carne ovina.

Desta forma, os atributos sensoriais da carne adquirem especial importância, sendo prioritários nas pesquisas ter como meta analisar a influência dos diferentes fatores sobre o produto final.

De acordo com Siqueira et al. (2002) o mercado de carne ovina no Brasil expandese de forma significativa nos últimos tempos. Entretanto, interpõe-se a um grave problema, devendo ser minimizado urgentemente: a qualidade do produto.

O processo de maturação apresenta influência nas propriedades organolépticas da carne, em especial na sua maciez e odor, influindo significativamente em sua pala- tabilidade (Pardi et al., 2001).

De acordo com Moura (1997) embora a utilização do cloreto de cálcio apresente vantagens no amaciamento da carne, sua adição pode resultar no aparecimento de um sabor amargo que pode ter efeito adverso sobre os outros atributos sensoriais da carne, fazendo com que o produto seja rejeitado pelo consumidor (Beekman, 1995, Pérez et al., 1998). Entretanto, Boleman et al. (1995) relataram que a adição de solução de $0,3 \mathrm{M}$ de cloreto de cálcio à carne bovina na proporção de $10 \%$ do peso da amostra foi efetiva na melhoria da maciez, mantendo a aceitabilidade da carne.

Segundo Rota (2005) embora estudos sobre a qualidade da carne ovina sejam amplamente descritos, existem poucos trabalhos relacionados às características sensoriais da carne de cordeiro no Brasil.

Sendo assim, este trabalho objetivou estudar os efeitos de diferentes períodos de maturação, da injeção com cloreto de cálcio e da interação desses fatores sobre as características sensoriais da carne de cordeiros Morada Nova proveniente dos músculos Biceps femoris (patinho), Longissimus e Triceps brachii (coração da paleta).

\section{MATERIALEMÉTODOS}

O experimento foi realizado na Faculdade de Ciências Agrárias e Veterinárias - FCAV/ UNESP, Jaboticabal-SP. A fase de campo foi desenvolvida nas dependências do Setor de Equinocultura, localizado no Centro de Pesquisas em Sanidade Animal (CPPAR), pertencente ao Departamento de Patologia Veterinária, a fase de abate dos cordeiros e refrigeração das carcaças, no setor de Ovinocultura, pertencente ao Departamento de Zootecnia, e a fase de análises físicas e químicas no Laboratório de Tecnologia dos Produtos de Origem Animal, pertencente ao Departamento de Tecnologia.

Foram utilizados 24 cordeiros da raça Morada Nova, machos inteiros, com peso vivo médio inicial de $15 \mathrm{~kg}$ (3 meses), os 
quais permaneceram alojados em galpão de confinamento até o abate, em baias coletivas de $12 \mathrm{~m}^{2}$ cada, compostas por 6 cordeiros, totalizando 4 baias equipadas com comedouros e bebedouros.

Os cordeiros foram identificados através de brincos e antes de entrarem na fase experimental receberam anti-helmíntico, vitamina ADE e ferro intramuscular.

A dieta experimental formulada de acordo com o NRC (1985) teve como volumoso feno de capim coastcross moído, e como concentrado protéico farelo de soja $(44,57 \%)$, concentrado energético milho moído (54,27\%), além de sal iodado $(0,61 \%)$ e núcleo mineral ovino $(0,55 \%)$.

A refeição foi fornecida no período matutino, sendo composta pela relação volumoso: concentrado de 40: 60. O acesso dos animais à alimentação foi livre, permitindo $10 \%$ de sobras nos comedouros.

Nos ingredientes da dieta experimental, foram determinados matéria seca, proteína bruta, extrato etéreo e matéria mineral, conforme metodologias descritas por Silva e Queiroz (2002). A composição dos ingredientes e da dieta experimental encontramse na tabela $\mathbf{I}$.

O consumo de matéria seca por cordeiro foi em média de $800 \mathrm{~g} /$ dia e o ganho médio de peso diário foi de $170 \mathrm{~g}$, originando uma

Tabela I. Composição (\% MS*) dos ingredientes (volumoso e concentrado) e da dieta experimental. (Composition (\% DM) of ingredients (roughage and concentrate) and experimental diet)

\begin{tabular}{lcccc}
\hline Ingrediente & $\mathrm{MS} \%$ & $\mathrm{~PB}^{*}$ & $\mathrm{EE}^{*}$ & $\mathrm{MM}^{*}$ \\
\hline Volumoso & 93,01 & 9,66 & 0,87 & 5,01 \\
Concentrado & 87,19 & 21,67 & 0,56 & 6,91 \\
Dieta experimental & 89,51 & 16,86 & 0,69 & 6,15 \\
EPM & 0,68 & 0,45 & 0,37 & 0,71
\end{tabular}

MS: matéria seca, PB: proteína bruta, EE: extrato etéreo, MM: matéria mineral, EPM: erro padrão da média.
Tabela II. Médias de pesos e características da carcaça de cordeiros Morada Nova $(n=24)$ abatidos aos 5 meses de idade. (Weights and carcass characteristics from Morada Nova lambs slaughtered at 5 months).

\begin{tabular}{lcc}
\hline Variável & Peso e característica & EPM \\
\hline PV $(\mathrm{kg})$ & 26,90 & 0,59 \\
PA $(\mathrm{kg})$ & 24,91 & 0,57 \\
PJ $(\%)$ & 7,45 & 0,61 \\
PCQ $(\mathrm{kg})$ & 11,49 & 0,48 \\
PCF $(\mathrm{kg})$ & 11,09 & 0,45 \\
QR $(\%)$ & 3,47 & 0,33 \\
RCQ $(\%)$ & 46,16 & 0,42 \\
RCF $(\%)$ & 44,56 & 0,44 \\
\hline
\end{tabular}

PV: peso vivo, PA: peso ao abate, PJ: perda de peso após o jejum, PCQ: peso da carcaça quente, PCF: peso da carcaça fria, QR: quebra ao resfriamento, RCQ: rendimento de carcaça quente, RCF: rendimento de carcaça fria, EPM: erro padrão da média.

conversão alimentar média de 4,71.

Os cordeiros foram abatidos ao atingirem $25 \mathrm{~kg}$ de peso vivo, em média aos 5 meses de idade. $O$ abate foi realizado após jejum de 16 horas de dietas hídrica e sólida. Após este período os cordeiros foram insensibilizados por meio de descarga elétrica de $220 \mathrm{~V}$ por 8 segundos, e em seguida seccionadas as veias jugulares e as artérias carótidas para a sangria. Na tabela II estão citados alguns parâmetros quantitativos observados durante o período experimental.

Após o abate, as carcaças permaneceram em câmara fria $4^{\circ} \mathrm{C}$ por 24 horas. Após este período os músculos (do lado direito da carcaça) Biceps femoris, Longissimus e Triceps brachii foram retirados para serem submetidos aos tratamentos. As carnes submetidas ao processo de maturação foram embaladas à vácuo e permaneceram em câmara fria durante 7 ou 14 dias a temperatura de $4^{\circ} \mathrm{C}$. A solução de cloreto de cálcio $0,3 \mathrm{M}$ foi preparada com água destilada e injetada na carne na proporção de $10 \%$ do peso da peça (Koohmaraie et al., 1990, 
Boleman et al., 1995, Moura et al., 1996, Moura et al., 1999, Fernandes, 2000, Polidori et al., 2000, Heinemann e Pinto, 2003, Jaturasitha et al., 2004).

Posteriormente foi realizada a análise sensorial conforme Moraes (1993). As amostras foram assadas em forno a gás, préaquecido a $170^{\circ} \mathrm{C}$, até que a temperatura interna da carne atingisse $75^{\circ} \mathrm{C}$. Foram então, cortadas e servidas a cada provador, em cabine individual, em recipientes plásticos codificados com três dígitos referentes aos respectivos tratamentos. Para a avaliação sensorial foram utilizados no mínimo 30 provadores não-treinados. Foi utilizada uma escala hedônica não-estruturada em 9 pontos, empregados para obter os seguintes atributos: cor, sabor (sensação de gosto e odor liberados pela amostra durante a mastigação), maciez (percepção da força necessária para cisalhar a amostra através da mordida) e impressão global (somatório de todas as percepções sensoriais expressando o exame dos julgadores sobre a qualidade do produto). Os 9 pontos da escala consistíram em: 1- desgostei muitíssimo, 2- desgostei muito, 3- desgostei regularmente, 4 - desgostei ligeiramente, 5 -indiferente, 6- gostei ligeiramente, 7- gostei regularmente, 8- gostei muito e 9- gostei muitíssimo (Moraes, 1993).

Para o músculo Longissimus o delineamento experimental utilizado foi em blocos casualizados, analisando-se os fatores (maturação e cloreto de cálcio) separadamente. Inicialmente verficou-se as possíveis diferenças na cor, sabor, maciez e impressão global nos diferentes tempos de maturação (0, 7 e 14 dias) nas carnes sem cloreto de cálcio (período 1 - foram fornecidas 3 amostras para cada provador), após comparou-se os mesmos atributos nos diferentes tempos de maturação (0, 7 e 14 dias) nas carnes com $0,3 \mathrm{M}$ de cloreto de cálcio (período 2 - foram fornecidas 3 amostras para cada provador). A melhor resposta do período 1 foi comparada com a melhor resposta do período 2. Esta metodologia foi utilizada pois, inicialmente o fornecimento de 6 amostras por provador foi considerado um número alto, entretanto, percebeu-se ao longo do experimento que 6 amostras por provador seria adequado, desta forma para os outros dois músculos comparou-se os 6 tratamentos de uma só vez.

Sendo assim o delineamento experimental utilizado para os músculos Biceps femoris e Triceps brachii foi em blocos casualizados, em esquema fatorial $3 \times 2$, totalizando 6 tratamentos e 4 blocos, sendo os fatores avaliados: o tempo de maturação (0, 7 e 14 dias) e a injeção com cloreto de cálcio (0 e $0,3 \mathrm{M})$.

Os tratamentos consistíram em: controle, maturação durante 7 dias, maturação durante 14 dias, cloreto de cálcio $0,3 \mathrm{M}$, maturação durante 7 dias e cloreto de cálcio $0,3 \mathrm{M}$, maturação por 14 dias e cloreto de cálcio $0,3 \mathrm{M}$.

Todas as análises de variância foram realizadas de acordo com o SAS (1996) e as comparações dos contrastes entre as médias dos tratamentos foram feitas pelo teste de Tukey a $5 \%$.

\section{RESULTADOSEDISCUSSÃO}

Na tabela III está apresentada a avaliação sensorial da carne de cordeiros Morada Nova proveniente do músculo Biceps femoris.

A análise de variância indicou que o tempo de maturação não influenciou $(p>0,05)$ os atributos sabor e maciez da carne, com médias de 6,71 e 7,13 , respectivamente. Entretanto, Borges et al. (2005) avaliando a maciez subjetiva da carne de caprinos proveniente dos músculos Longissimus dorsi, Semimembranosus e Biceps femoris, observaram que as carnes maturadas foram mais macias em relação àquelas que não foram maturadas.

$O$ fator cloreto de cálcio também não afetou $(p>0,05)$ estes atributos, apresentando valores de 6,71 e 7,13, para o sabor e a maciez, respectivamente. Todavia, Miller 
et al. (1995) ao estudarem o efeito da concentração de cloreto de cálcio na maciez subjetiva da carne bovina, observaram que o painel de provadores atribuiu maior maciez $(5,9)$ à carne que recebeu $0,2 \mathrm{M}$ de cloreto de cálcio, em comparação às carnes do tratamento controle $(5,4)$.

De acordo com Moura (1997) embora a utilização do cloreto de cálcio apresente vantagens no amaciamento da carne, sua adição pode resultar no aparecimento de sabor amargo que pode ter efeito adverso sobre os outros atributos sensoriais da carne, fazendo com que o produto seja rejeitado pelo consumidor (Beekman, 1995, Pérez et $a l ., 1998)$, entretanto este fato não foi observado nesta pesquisa. Morgan et al. (1991) observaram o desenvolvimento de sabor amargo e metálico em carne bovina injetada com solução $0,3 \mathrm{M}$ de cloreto de cálcio.

Tabela III. Avaliação sensorial da carne proveniente do músculo Biceps femoris $(n=24)$ em cordeiros da raça Morada Nova nos diferentes tempos de maturação e nas diferentes concentrações de cloreto de cálcio. (Sensorial evaluation of meat from Biceps femoris muscle in Morada Nova lambs in different times of ageing and calcium chloride concentrations).

\begin{tabular}{|c|c|c|}
\hline Maturação (días) & Sabor & Maciez \\
\hline 0 & 6,6 & 7,0 \\
\hline 7 & 6,9 & 7,2 \\
\hline 14 & 6,6 & 7,1 \\
\hline $\mathrm{p}$ & NS & NS \\
\hline EPM & 0,30 & 0,24 \\
\hline \multicolumn{3}{|l|}{$\mathrm{CaCl}_{2}$} \\
\hline 0 & 6,6 & 6,9 \\
\hline $0,3 \mathrm{M}$ & 6,8 & 7,3 \\
\hline $\mathrm{p}$ & NS & NS \\
\hline EPM & 0,30 & 0,24 \\
\hline Teste $\mathrm{F}$ para int. $\mathrm{M} \times \mathrm{C}$ & 5,05 & 1,21 \\
\hline $\mathrm{p}$ & $<0,05$ & NS \\
\hline $\mathrm{CV}(\%)$ & 8,13 & 6,90 \\
\hline
\end{tabular}

p: probabilidade, EPM: erro padrão da média, CV: coeficiente de variação.
Tabela IV. Desdobramento da interação $M$ $x C$ (tempos de maturação e concentrações de cloreto de cálcio) para o atributo sabor da carne proveniente do músculo Biceps femoris $(n=24)$ em cordeiros da raça Morada Nova. (Interaction $\mathrm{M} \times \mathrm{C}$ (time of ageing and calcium chloride concentrations) for flavor attribute coming from Biceps femoris muscle in Morada Nova lambs).

\begin{tabular}{|c|c|c|c|c|c|}
\hline \multicolumn{6}{|c|}{ días } \\
\hline Maturação & 0 & 7 & 14 & Teste F & $p$ \\
\hline Sem CaCl & $6,7^{\text {Aa }}$ & $7,0^{\mathrm{Aa}}$ & $6,0^{\mathrm{Ba}}$ & 3,64 & $<0,05$ \\
\hline $0,3 \mathrm{M} \mathrm{CaCl}_{2}$ & $6,5^{\mathrm{Aa}}$ & $6,7^{\text {Aa }}$ & $7,2^{\mathrm{Aa}}$ & 1,96 & NS \\
\hline Teste F & 0,42 & 0,42 & 10,51 & - & - \\
\hline $\mathrm{p}$ & NS & NS & NS & - & - \\
\hline EPM & 0,30 & 0,30 & 0,30 & - & - \\
\hline
\end{tabular}

a,bmédias seguidas por letras distintas na coluna, diferem entre si pelo teste de Tukey.

$\mathrm{A}, \mathrm{B}$ médias seguidas por letras distintas na linha, diferem pelo teste de Tukey.

p: probabilidade, EPM: erro padrão da média.

A interação entre os fatores foi não significativa $(\mathrm{p}>0,05)$ para o atributo maciez, entretanto foi significativa a $5 \%$ de probabilidade para o atributo sabor.

Madruga et al. (2005) ao realizarem análise sensorial da carne proveniente da perna de cordeiros Santa Inês, observaram valores atribuídos à maciez de 7,17 a 7,85, os quais foram próximos aos desta pesquisa. Entretanto, para o atributo sabor os autores observaram valores superiores, de 7,05 a 7,65 .

A tabela IV apresenta o desdobramento da interação para o atributo sabor da carne de cordeiros Morada Nova.

Como pode ser observado na tabela IV o fator cloreto de cálcio não apresentou diferença significativa ( $p>0,01)$ para as carnes não maturadas, maturadas durante $7 \mathrm{e}$ 14 dias, com médias de 6,$63 ; 6,88$ e 6,63, respectivamente.

O fator tempo de maturação influenciou $(\mathrm{p}<0,01)$ as carnes sem cloreto de cálcio aos 14 dias de maturação. As carnes apre- 
Tabela $V$. Avaliação sensorial da carne proveniente do músculo Longissimus $(n=24) \mathrm{em}$ cordeiros da raça Morada Nova nos diferentes tempos de maturação e sem cloreto de cálcio. (Sensorial evaluation of meat from Longissimus muscle in Morada Nova lambs at differen times of ageing and without calcium chloride).

\begin{tabular}{ccccc}
\hline $\begin{array}{c}\text { Maturação } \\
\text { (dias) }\end{array}$ & Cor & Sabor & Maciez & $\begin{array}{c}\text { Impressão } \\
\text { global }\end{array}$ \\
\hline \multicolumn{1}{c}{0} & 7,0 & 7,5 & 7,5 & 7,2 \\
7 & 7,2 & 7,7 & 8,0 & 8,0 \\
14 & 7,0 & 7,0 & 8,0 & 7,5 \\
p & NS & NS & NS & NS \\
EPM & 0,19 & 0,47 & 0,11 & 0,25 \\
CV (\%) & 6,23 & 9,27 & 4,26 & 6,59 \\
\hline
\end{tabular}

p: probabilidade; EPM: erro padrão da média; CV: coeficiente de variação.

sentaram nota mais baixa $(6,00)$ em relação àquelas não maturadas $(6,75)$ e maturadas durante 7 dias $(7,00)$, as quais não diferiram entre si $(p>0,05)$. Para as carnes que receberam cloreto de cálcio não houve efeito $(\mathrm{p}>0,05)$ do tempo de maturação, com nota média de 6,83.

Na tabela $\mathbf{V}$ está apresentada a avaliação sensorial da carne de cordeiros Morada Nova proveniente do músculo Longissimus.

Conforme os resultados dispostos na tabela $\mathbf{V}$ pode-se observar que o tempo de maturação não afetou $(\mathrm{p}>0,05)$ os atributos sensoriais da carne sem cloreto de cálcio, com médias de 7,$08 ; 7,42 ; 7,83$ e 7,58 para a cor, o sabor, a maciez e a impressão global, respectivamente.

Rota (2005) estudando a maciez subjetiva da carne de cordeiros Corriedale abatidos aos 4 meses de idade, no músculo Longissimus dorsi, obteve valor inferior $(5,24)$ em relação ao valor deste experimento $(7,50)$. Segundo Sañudo (2002), valores crescentes ou decrescentes na maciez da carne podem ser encontrados em animais jovens, talvez em função de interações entre diversos fatores, entre eles a deposição de gordura intramuscular. Entretanto, o valor observado por Rota (2005) está próximo ao valor (5,32) observado por Sá et al. (2004) ao avaliarem, no músculo Longissimus lumborum, a maciez subjetiva de cordeiros Santa Inês abatidos aos $31 \mathrm{~kg}$ de peso vivo.

Estes autores também observaram subjetivamente a cor e o sabor das carnes de cordeiros Santa Inês e Hampshire Down, com valores de 6,61 e 6,72 para a raça Santa Inês e 6,19 e 6,84 para a raça Hampshire Down, valores inferiores aos desta pesquisa.

Siqueira et al. (2002) avaliando caracteres sensoriais da carne de cordeiros Hampshire Down, Santa Inês e Bergamácia $\mathrm{x}$ Corriedale, proveniente do músculo Longissimus dorsi, observaram para o atributo sabor valores de 6,$9 ; 6,6$ e 7,4, respectivamente, já para o atributo maciez, obtiveram valores de 5, $0 ; 5,0$ e 3,4, considerados inferiores aos deste experimento.

Para avaliar a maciez subjetiva da carne (Longissimus dorsi) de cordeiros Ile de France x Texel criados em confinamento e abatidos aos $28 \mathrm{~kg}$, Frescura et al. (2005) observaram valor para a maciez de 7,37 , valor próximo ao deste experimento para carnes não maturadas e sem cloreto de cálcio.

Tabela VI. Avaliação sensorial da carne proveniente do músculo Longissimus $(n=24)$ em cordeiros da raça Morada Nova nos diferentes tempos de maturação e na concentração 0,3 M de cloreto de cálcio. (Sensorial evaluation of meat from Longissimus muscle in Morada Nova lambs at different times of ageing and calcium chloride concentration 0.3 M).

\begin{tabular}{ccccc}
\hline $\begin{array}{c}\text { Maturação } \\
\text { (dias) }\end{array}$ & Cor & Sabor & Maciez & $\begin{array}{c}\text { Impressão } \\
\text { global }\end{array}$ \\
\hline \multicolumn{1}{c}{0} & 7,0 & 7,7 & 7,7 & 7,7 \\
7 & 7,0 & 7,2 & 7,7 & 7,7 \\
$\quad 14$ & 7,0 & 7,7 & 8,0 & 8,0 \\
p & - & NS & NS & NS \\
EPM & 0 & 0,44 & 0,08 & 0,08 \\
CV (\%) & 0 & 8,79 & 3,69 & 3,69 \\
\hline
\end{tabular}

p: probabilidade; EPM: erro padrão da média; CV: coeficiente de variação. 
Tabela VII. Avaliação sensorial da carne proveniente do músculo Longissimus $(n=24)$ em cordeiros da raça Morada Nova nas diferentes concentrações de cloreto de cálcio e no tempo de maturação durante 14 dias. (Sensorial evaluation of meat from Longissimus muscle in Morada Nova lambs in different calcium chloride concentrations aged for 14 days).

\begin{tabular}{lcccc}
\hline $\mathrm{CaCl}_{2}$ & Cor & Sabor & Maciez & $\begin{array}{c}\text { Impressão } \\
\text { global }\end{array}$ \\
\hline 0 M & 7,5 & 7,5 & 7,7 & 7,7 \\
$0,3 \mathrm{M}$ & 7,2 & 8,0 & 8,0 & 7,7 \\
$\mathrm{p}$ & $\mathrm{NS}$ & $\mathrm{NS}$ & $\mathrm{NS}$ & $\mathrm{NS}$ \\
$\mathrm{EPM}$ & 0,35 & 0,40 & 0,35 & 0,57 \\
$\mathrm{CV}(\%)$ & 4,79 & 5,27 & 4,49 & 7,45 \\
\hline
\end{tabular}

p: probabilidade; EPM: erro padrão da média; $C V$ : coeficiente de variação.

Os valores médios encontrados na maciez sensorial da carne estão superiores aos citados por Suarez et al. (2000), que obtiveram para carne não maturada de cordeiros Corriedale, valores de 5,2 para o sabor e 6,2 para a maciez.

Na tabela VI está apresentada a avaliação sensorial da carne de cordeiros Morada Nova proveniente do músculo Longissimus.

De acordo com os resultados apresentados na tabela VI pode-se observar que o tempo de maturação não afetou $(p>0,05)$ os atributos sensoriais da carne com cloreto de cálcio, com médias de 7,08; 7,42; 7,83 e 7,58 para a cor, o sabor, a maciez e a impressão global, respectivamente. Entretanto, Hoover et al. (1995) ao avaliar a maciez subjetiva da carne bovina e a intensidade de sabor, observaram que as carnes tratadas com $0,2 \mathrm{M}$ de cloreto de cálcio apresentaram nota maior pelos provadores em relação às amostras do tratamento controle.

Na tabela VII está apresentada a avaliação sensorial da carne de cordeiros Morada Nova proveniente do músculo Longissimus.

Do mesmo modo que nas tabelas V e VI, na tabela VII pode ser observado que não houve efeito significativo $(p>0,05)$ da concentração de cloreto de cálcio para as carnes maturadas durante 14 dias para os atributos cor, sabor, maciez e impressão global, com médias de 7,38; 7,75; 7,88 e 7,75, respectivamente. Igualmente, avaliando o efeito de diferentes concentrações de cloreto de cálcio $(0,2$ e $0,25 \mathrm{M})$ na qualidade da carne de bovinos, Wheeler et al. (1993) não observaram diferenças no sabor e na suculência da carne injetada com cloreto de cálcio.

Boleman et al. (1995) relataram que a adição de solução de $0,3 \mathrm{M}$ de cloreto de cálcio à carne bovina na proporção de $10 \%$ do peso da amostra foi efetiva na melhoria da maciez, mantendo a aceitabilidade da carne.

$\mathrm{Na}$ tabela VIII está apresentada a avaliação sensorial da carne de cordeiros Morada Nova proveniente do músculo Triceps brachii.

Tabela VIII. Avaliação sensorial da carne proveniente do músculo Triceps brachii $(n=24)$ em cordeiros da raça Morada Nova nos diferentes tempos de maturação e nas diferentes concentrações de cloreto de cálcio. (Sensorial evaluation of meat from Triceps brachii muscle in Morada Nova lambs at different times of ageing and calcium chloride concentrations).

\begin{tabular}{|c|c|c|}
\hline $\begin{array}{l}\text { Maturação } \\
\text { días }\end{array}$ & Sabor & Maciez \\
\hline 0 & 7,1 & 7,1 \\
\hline 7 & 7,1 & 7,6 \\
\hline 14 & 6,4 & 7,2 \\
\hline $\mathrm{P}$ & NS & NS \\
\hline EPM & 0,45 & 0,60 \\
\hline \multicolumn{3}{|l|}{$\mathrm{CaCL}_{2}$} \\
\hline $0^{2}$ & 7,1 & 7,4 \\
\hline $0,3 \mathrm{M}$ & 6,7 & 7,2 \\
\hline$p$ & 0,1501 & 0,6059 \\
\hline EPM & 0,45 & 0,60 \\
\hline Teste $\mathrm{F}$ para int. $\mathrm{M} \times \mathrm{C}$ & 0,37 & 0,07 \\
\hline $\mathrm{p}$ & 0,6981 & 0,9332 \\
\hline CV (\%) & NS & NS \\
\hline
\end{tabular}

p: probabilidade; EPM: erro padrão da média; CV: coeficiente de variação. 
A análise de variância da tabela VIII indicou que o tempo de maturação não influenciou $(\mathrm{p}>0,05)$ os atributos sabor e maciez da carne, com médias de 6,88 e 7,34, respectivamente. $\mathrm{O}$ fator cloreto de cálcio também não afetou $(p>0,05)$ estes atributos, apresentando valores de 6,88 e 7,34, para o sabor e a maciez, respectivamente. Entretanto, Heinemann e Pinto (2003) estudando o efeito de diferentes concentrações de cloreto de cálcio $(0 ; 0,1 ; 0,2$ e $0,3 \mathrm{M})$ na aceitabilidade da carne bovina maturada, observaram que a maioria dos provadores (67\%) consideraram as amostras tratadas com cloreto de cálcio mais macias do que as não tratadas.

Fernandes (2000) ao avaliar atributos sensoriais da carne bovina (músculo Longissimus dorsi) observou que não houve diferença significativa $(p>0,05)$ entre as amostras tratadas e não tratadas com cloreto de cálcio no que se refere aos atributos maciez e suculência, embora em relação ao sabor e a qualidade global, o resulto tenha

\section{BIBLIOGRAFIA}

Beekman, D.D. 1995. The effects of calcium chloride, sodium chloride, and tripolyphosphate injections on tenderness, water holding capacity and microscopic structure of beef muscles. Diss. Abs. Int., 55: 156-175.

Boleman, S.J., Boleman, S.L., Bidner, T.D., Mcmillin, K.W. and Monlezun, C.J. 1995. Effects of post mortem time of calcium chloride injection on beef tenderness and drip, cooking, and total loss. Meat Sci., 39: 35-41.

Borges, A.S., Zapata, J.F.F., Garruti, D.S., Rodrigues, M.C.P., Freitas, E.R. e Pereira, A.L.F. 2005. Correlação entre as medições instrumentais e sensoriais da dureza e suculência da carne caprina. Em: Congresso Brasileiro de Ciência e Tecnologia de Carnes, 3 , 2005. São Pedro. Anais... São Pedro.

Fernandes, J.R. 2000. Avaliação de diferentes tecnologias para o amaciamento da carne bovina in natura. Dissertação. Faculdade de Engenharia de Alimentos. Universidade Estadual de Campinas. Campinas. Brasil. 150 pp. sido o oposto. Segundo o autor esta constatação permite concluir que a aplicação de cloreto de cálcio tornou a carne do experimento imprória para o consumo, apesar da maciez observada. Nesta pesquisa os provadores não relataram problemas quanto ao sabor das amostras avaliadas.

Constatou-se que a carne dos cordeiros, apresentou valores característicos de elevada qualidade e aceitabilidade, pois de acordo com Siqueira et al. (2002) valores da escala hedônica não estruturada entre 6,1 e 7,4 representam carne de alta qualidade.

A interação entre os fatores foi não significativa $(\mathrm{p}>0,05)$ para os atributos sabor e maciez.

\section{CONCLUSÕES}

A qualidade sensorial da carne de cordeiros Morada Nova, mostrou-se semelhante quanto aos atributos avaliados quando foi submetida aos processos de maturação e injeção com cloreto de cálcio.

Frescura, R.B.M., Pires, C.C., Silva, J.H.S., Müller, L., Cardoso, A., Kippert, C.J., Peres Neto, D., Silveira, C.D., Alebrante, L. e Thomas, L. 2005. Avaliação das proporções dos cortes da carcaça, características da carne e avaliação dos componentes do peso vivo de cordeiros. Rev. Bras. Zootecn., 34: 167-174.

Heinemann, R.J.B. e Pinto, M.F. 2003. Efeito da injeção de diferentes concentrações de cloreto de cálcio na textura e aceitabilidade de carne bovina maturada. Ciênc. Tecnol. Aliment., 23: 146-150.

Hoover, L.C., Cook, K.D., Miller, M.F., Huffman, K.L., Wu, C.K., Lansdell, J.L. and Ramsey, C.B. 1995. Restaurant consumer acceptance of beef loin strip steaks tenderized with calcium chloride. J. Anim. Sci., 73: 3633-3638.

Jaturasitha, S., Thirawong, P., Leangwunta, V. and Kreuzer, M. 2004. Reducing toughness of beef from Bos indicus draught steers by injection of calcium chloride. Effect of concentration and time post mortem. Meat Sci., 68: 61-69. 


\section{CARACTERÍSTICAS DA CARNE DE CORDEIRO MATURADA E INJETADA COM CL ${ }_{2}$ CA}

Koohmaraie, M., Whipple, G. and Crouse, J.D. 1990. Acceleration of post mortem tenderization in lamb and Brahman-cross beef carcasses through infusion of calcium chloride. J. Anim. Sci., 68: 1278-1283.

Madruga, M.S., Sousa, W.H., Rosales, M.D., Cunha, M.G.G. e Ramos, J.L.F. 2005. Qualidade da carne de cordeiros Santa Inês terminados com diferentes dietas. Rev. Bras. Zootecn., 34: 309315.

Martínez-Cerezo, S., Sañudo, C., Panea, B. and Olleta, J.L. 2005. Breed, slaughter weight and ageing time effects on consumer appraisal of three muscles of lamb. Meat Sci., 69: 795-805.

Miller, M.F., Huffman, K.L., Gilbert, S.Y., Hamman, L.L. and Ramsey, C.B. 1995. Retail consumer acceptance of beef tenderized with calcim chloride. J. Anim. Sci., 73: 2308-2314.

Moraes, M.A.C. 1993. Métodos para avaliação sensorial dos alimentos. Editora da Unicamp. Campinas, SP. 93 pp.

Morgan, J.B., Miller, R.K., Mendes, F.M., Hole, D.S. and Savell, J.W. 1991. Using calcium chloride injection to improve tenderness of beef from mature cows. J. Anim. Sci., 69: 4469-4476.

Moura, A.C., Luchiari Filho, A., Landaeta, F.A.C. e Zinsly, C.F. 1996. Estudo da utilização de cloreto de cálcio no amaciamento da carne de coelho através do processo de marinação. Em: Reunião Anual da Sociedade Brasileira de Zootecnia, 35, 1996. Fortaleza. Anais... Sociedade Brasileira de Zootecnia. Fortaleza. pp. 560-562.

Moura, A.C. 1997. Efeito da injeção pós-morte de cloreto de cálcio e tempo de maturação, no amaciamento e perdas por cozimento do músculo Longissimus dorsi de animais Bos indicus e Bos taurus selecionados para ganho de peso. Dissertação. Escola Superior de Agricultura Luiz de Queiroz. Universidade de São Paulo. Piracicaba. 78 pp.

Moura, A.C., Luchiari Filho, A., Nardon, R.F. e Razook, A.G. 1999. Efeitos da injeção de cloreto de cálcio pós-morte e tempo de maturação no amaciamento e nas perdas por cozimento do músculo Longissimus dorsi de animais Bos indicus e Bos taurus selecionados para ganho de peso. Rev. Bras. Zootecn., 28: 1382-1389.

NRC. 1985. National Research Council. Nutrient requirements of sheep. 1985. National Academy Press. Washington, NY. 99 pp.
Osório, J.C.S., Osório, M.T.M., Jardim, P.O.C. Pimentel, M.A., Pouey, J.L.O., Lüder, W.E. Cardellino, R.A., Oliveira, N.M., Gularte, M.A., Borba, M.F., Motta, L., Esteves, R., Monteiro, E. e Zambiazi, R. 1998. Métodos para avaliação da produção de carne ovina: in vivo, na carcaça e na carne. Universidade Federal de Pelotas. Pelotas, RS. 107 pp.

Pardi, M.C., Santos, I.F., Souza, E.R. e Pardi, H.S. 2001. Ciência, higiene e tecnologia da carne. $2^{\mathrm{a}}$ ed. Universidade Federal de Goiânia. Goiânia, GO. 623 pp.

Pérez, M.L., Escalona, H. and Guerrero, I. 1998. Effect of calcium chloride marination on calpain and quality characteristics of meat from chicken, horse, cattle and rabbit. Meat Sci., 48: 125-134.

Polidori, P., Marinucci, M.T., Fantuz, F., Renieri, C. and Polidori, F. 2000. Tenderization of wether lambs meat through pre-rigor infusion of calcium ions. Meat Sci., 55: 197-200.

Rota, E.L. 2005. Efeito da castração e idade de abate sobre a qualidade da carne em cordeiros da raça Corriedale. Tese. Faculdade de Agronomia Eliseu Maciel. Universidade Federal de Pelotas. Pelotas. $88 \mathrm{pp}$.

Sá, J.L., Siqueira, E.R., Roça, R.O., Sá, C.O. e Fernandes, S. 2004. Características sensoriais da carcaça de cordeiros Hampshire Down e Santa Inês submetidos a dois fotoperíodos na fase de terminação em confinamento. Em: Reunião Anual da Sociedade Brasileira de Zootecnia, 41, 2004. Campo Grande. Anais... Sociedade Brasileira de Zootecnia. Campo Grande. pp. 1-5.

Sañudo, C. 2002. Factors affecting carcass and meat quality in lambs. Em: Reunião Anual da Sociedade Brasileira de Zootecnia, 39, 2002. Recife. Anais... Sociedade Brasileira de Zootecnia. Recife. pp. 434-455.

SAS. 1996. User's Guide to Statistics. Version 6.12. Cary. North Caroline State University. USA.

Silva, D.J. e Queiroz, A.C. 2002. Análise de alimentos: métodos químicos e biológicos. $3^{\mathrm{a}} \mathrm{ed}$. UFV. Viçosa, MG. 235 pp.

Silva, M.A.A.P. 2002. Métodos de avaliação sensorial dos alimentos. Em: Curso de Extensão, 2002. Anais... Campinas. Faculdade de Engenharia de Alimentos. Campinas. 73 pp.

Siqueira, E.R., Roça, R.O., Fernandes, S. e Uemi, 


\section{ZEOLA, SOUZA, SOUZA E SILVA SOBRINHO}

A. 2002. Características sensoriais da carne de cordeiros das raças HampshireDown, Santa Inês e Mestiços Bergamácia x Corriedale abatidos com quatro distintos pesos. Rev. Bras. Zootecn., 31: 1269-1272.

Suarez, L.V., Busetti, M.R., Garriz, C.A., Gallinger, M.M. and Babinec, F.J. 2000. Pre-weaning growth, carcass traits and sensory evaluation of Corriedale, Corriedale x Pampinta and Pampinta lambs. Small Ruminant Res., 36: 85-89.
Vilas Boas, A.S. 2005. Como obter carne ovina de qualidade para expansão do consumo. Em: Congresso Brasileiro de Ciência e Tecnologia de Carnes, 3, 2005. São Pedro. Anais... São Pedro.

Wheeler, T.L., Koohmaraie, M., Lansdell, J.L., Siragusa, G.R. and Miller, M.F. 1993. Effects of post mortem injection time, injection level, and concentration of calcium chloride on beef quality traits. J. Anim. Sci., 71: 2965-2974. 